\title{
Téoros
}

Revue de recherche en tourisme

\section{Mise en valeur du Saint-Laurent}

Le rôle du tourisme

\section{Hugues Morrissette}

Volume 6, numéro 2, juillet 1987

Le Saint-Laurent magnétique

URI : https://id.erudit.org/iderudit/1080504ar

DOI : https://doi.org/10.7202/1080504ar

Aller au sommaire du numéro

Éditeur(s)

Université du Québec à Montréal

ISSN

0712-8657 (imprimé)

1923-2705 (numérique)

Découvrir la revue

Citer cet article

Morrissette, H. (1987). Mise en valeur du Saint-Laurent : le rôle du tourisme.

Téoros, 6(2), 10-11. https://doi.org/10.7202/1080504ar d'utilisation que vous pouvez consulter en ligne.

https://apropos.erudit.org/fr/usagers/politique-dutilisation/ 


\title{
Le rôle du tourisme
}

\section{Mise en valeur du Saint-Laurent}

\author{
par Hugues Morrissette*
}

En juin 1985, le gouvernement du Québec rendait publique une stratégie de mise en valeur du Saint-Laurent.

Cette stratégie était retenue comme la politique officielle du gouvernement et, en suite logique de cette décision, fut créé le Secrétariat à la mise en valeur du Saint-Laurent. Le nouveau gouvernement, élu en décembre 1985, reconduisait le mandat du Secretariat. Constitué d'une équipe légère, il a pour mandat d'en favoriser la mise en valeur ordonnée de tous les eléments susceptibles d'en favoriser le développement economique. Ceci, évidemment, en harmonie avec les autres volets du strict développement Economique. En effet, il doit prendre en compte les préoccupations sociales, environnementales, etc.

Lors des travaux préalables à la confection du rapport du Projet Saint-Laurent, il est devenu rapidement évident qu'un besoin de concertation entre tous les acteurs etait absolument vital pour atteindre cet objectif. Un des volets importants évoqués au cours des diverses consultations était celui d'un relatif sous-développement de la dynamique référant à la mise en valeur du potentiel touristique et récréatif par cet atout économique dont dispose le Québec: LE SAINT-LAURENT.

Plusieurs mémoires ont fait état de la faiblesse des infrastructures $d^{+}$accueil ou d'opération dans certaines régions. D'autres ont noté que la publicité faite autour des atouts existants offerts par le Saint-Laurent était faible, lorsqu'elle existait.

À la décharge des responsables de cette situation, on doit avouer que, jusqu'à tout recemment, le Saint-Laurent n'avait pas fait l'objet d'une prise de conscience nationale comme c'est le cas depuis quelques années. Pour beaucoup de gens, le Saint-Laurent

\footnotetext{
- Monsieur Hugues Morriesette est secrétaine gotndral associd au Secretariat a la mise en valeur du SaintLaurent. Les wes que M. Morriesette exprima dans oet article ne reprtaentant pas forcément celles du gouwemament du Quebec.
}

était un obstacle qui, au surplus, était de juridiction fédérale. D'autres y voyaient une source d'embêtements: déglaçage durant l'hiver, pose et enlèvement des bouées, dragage, nécessitế de construire des ponts fort coutteux, de maintenir un service complet de traversiers, etc. $\hat{A}$ la limite, certains auraient préféré ne pas en avoir du tout ou encore, de le remplir et de le paver!

Surimposée à ces attitudes parfois navrantes, la multiplicité des juridictions et des acteurs ayant voix a la mise en valeur du Saint-Laurent $n^{\prime}$ a jamais facilité une action commune concertéc. D'où une prise de conscience organisée relativement jeune. En conséquence, il est compréhensible que les actions concernant le Saint-Laurent aient étế peu nombreuses et toutes récentes.

Lors de la publication de son rapport, en juin 1985, le gouvernement du Québec affirmait:

Par nombre de traits, le Saint-Laurent et ses affuents constituent un potentiel récréatif et touristique tout à fait exceptionnel. Les eawx, leurs rives, les villes, villages et sites riverains, leurs habitans; la faune, la flore, les formations géologiques visibles, les forêts, les routes, l'architecture; les industries, les genres de vie, l'histoire, les sites archéologiques, les musées, les ports, la roponymie, les climats et les saisons, tout cela pourrait contribuer à faire de la voie laurentienne un trésor inépuisable pour les vacanciers. Il y en a pour les marins, pour les historiens, pour les cyclistes ou les campeurs, pour les passagers des divers moyens de transports, pour les amoureux de la nature, les sporifs et les curieux de tout ou de rien. Hôtels, pensions, terrains de camping, villes industrielles, lieut sauvages, ports de plaisance ou de péche pourraient alterner indefiniment sur rivières et fleuve. On ne peut compter les accupations, les genres d'itinéraires de vacances ou de tourisme awxquels peut donner lieu le SaintLaurent habité ou inhabité. Pour peu qu'on en sente l'attrait et qu'on y réserve le temps, le Saint-Laurent, mieux aménagé pour le tourisme, deviendrait accessible à un très grand nombre et aux coûts les plus acceptables. ${ }^{11)}$

L'ensemble du couloir du Saint-Laurent, y compris le Saguenay, le Richelieu et $l^{\prime}$ Outaouais, offre donc un potentiel exceptionnel pour la pratique d'activités de plein air de toutes sortes pendant la plupari des mois de l'année. Pour réaliser ce potentiel, il faut protéger et aménager les sites les plus propices, en faciliter l'accès aux usagers tout en tenant compte des besoins de conservation, ce qui suppose des aménagements physiques légers. Pour favoriser le nautisme, il faut des rampes de mise à l'eau, des ports de plaisance avec services et des refuges d'urgence.

Les services aux clients, tels que le transport et l'hébergement devraient le plus souvent être laissés à l'entreprise privée. Mais celle-ci ne pourrait vraisemblablement le faire sans une aide de l'État car les investissements de départ seraient rentables qu'a plus ou moins long terme dans certains cas.

\section{Les intervenants}

Dans le secteur de la récréation et du tourisme, comme dans les autres secteurs maritimes, les acteurs sont nombreux: gouvernement du Canada, gouvernement du Québec, municipalités régionales de comté (MRC), municipalités, organismes privés et individus. Au fédéral, Parcs Canada, le Service canadien de la faune et Tourisme Canada se préoccupent, dans le cadre de leurs mandats, de la conservation et de la mise en valeur de sites remarquables transformés en parcs ou en réserves fauniques.

Au Québec, la récréation de plein air et la conservation de la faune relevent du Loisir, de la Chasse et de la Pêche (MLCP). Entre autres choses, le MLCP doit établir graduellement un réseau de parcs de conservation ou de récréation, ainsi qu'un réseau de réserves fauniques; il lui revient d'implanter une politique des services au public pour la pratique de ces activités de récréation. Le ministère peut agir directement ou déléguer ces tăches à des individus, organismes ou gouvernements locaux (concessionnaires, pourvoyeurs, ZEC, 
MRC, etc.). Mais cela n'empêche pas des interventions directes d'autres ministères. Le ministère de l'Environnement est intervenu à l'ìle Sainte-Thérèse; celui de l'Énergie et des Ressources administre plusieurs centres de nature, notamment ceux de Duchesnay et des Palissades dans Charlevoix. Quant au ministère des Affaires culturelles, il lui revient de voir à protéger et à mettre en valeur le patrimoine culturel: ses interventions touchent en majeure partie des lieux du couloir laurentien. A d'autres ministères encore, tel celui du Tourisme, il appartient de produire du matériel d'information ou de publicité et de s'occuper des infrastructures. Pour leur part, les municipalités et les MRC gèrent des services et des équipements provenant souvent des autres gouvernements. Enfin, nombre d'organismes privés ou d'individus offrent des services de récréation et de tourisme; les "zones d'exploitation contrólée" , les ports de plaisance et les entreprises d'excursions ou de croistères en sont des exemples.

La multiplicité d'intervenants n'est pas en soi troublante. Elle atteste même du grand nombre des intéressés. Toutefois, la cohérence des responsabilités n'est pas toujours servie par l'évolution empirique ou l'empiétement des champs d'action. On a constaté qué des projets intếressants s'étaient heurtés assez fréquemment ou bien aux incertitudes liees aux champs de responsabilité des nombreux intervenants ou bien à des difficultés administratives. Le Projet Saint-Laurent en est venu à souhaiter vivement que soit revue l'économie d'ensemble des intervenants en matière de récréation et de tourisme ou, à tout le moins, que soit facilitée la concertation des responsables, car il y va non seulement des projets dans ce secteur mais aussi de la possibilité d'améliorer le dialogué concernant et ce domaine et plusieurs autres qui touchent à la mise en valeur du Saint-Laurent.

Il y a probablement là un des problèmes majeurs de la mise en valeur du SaintLaurent; celui de perdre de vue la valeur et l'importance des actions complémentaires habituellement confiées à des réseaux différents.

Le Saint-Laurent a fait l'objet de nombreux inventaires et de nombreuses études, mais on s"est rarement penché sur des travaux de synthèse permettant de tirer profit des informatons dispersées et disparates. De la même façon, dans l'un ou l'autre des domaines du commerce, du patrimoine, de la faune, du tourisme, on a fait preuve d'initiative. Mais rarement a-t-on réussi à conjuguer les efforts entre les responsables, alors que la réalitế n'est pas aussi divisée

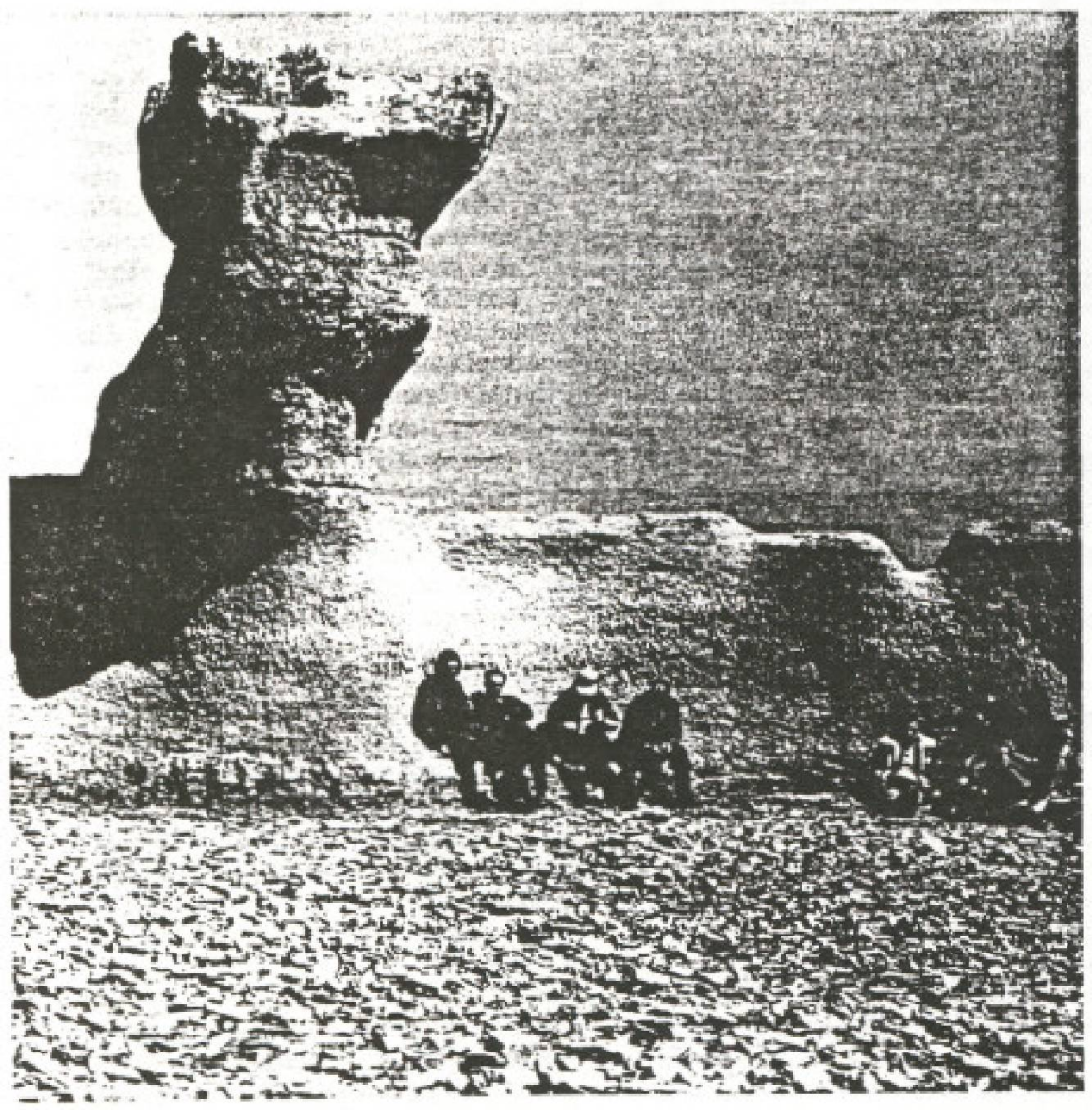

Pare national des lles Mingan que le sont les professionnels ou les spécialistes. Or, quand on parle de mise en valeur, on vise autant le progrès dans l'un ou l'autre domaine que la complémentarité et l'entraide dans le choix des solutions aux problemes; on vise autant le bénéfice dans chaque type d'activité que la satisfaction du plus grand nombre, quel que soit son champ d'intérêt. C'est dire qu'on ne peut négliger indùment la concertation des efforts sans s'exposer tôt ou tard à un rattrapage par trop onéreux. II n'est jamais possible de tout faire en meme temps. A force de se concentrer sur un seul aspect, on finit nécessairement par rendre extremement ardue la solution des problemes d'ensemble.

A mesure que le Projet Saint-Laurent a étendu ses investigations à d'autres domaines que celui du commerce maritime, non seulement a-t-il vu se multiplier les exemples de champs d'action où les concertations paraissaient faibles, mais encore a-t-il vu grandir les avantages d'êtablir des ponts entre les milieux intéressés. L'exploitation commerciale ne pourrait progresser longtemps si elle devait systématiquement ignorer les problèmes de la qualité des eaux; la récréation et le tourisme ont tout à gagner de la mise en valeur du patrimoine et d'une association avec les responsables des réseaux routier et ferroviaire, et vice versa.

Bien sûr, les juridictions gouvernementales multiples souvent coordonnées de façon empirique et aléatoire constituent-elles une difficulté majeure. Mais il y a fort à parier qu'une meilleure coordination au Québec apporterait déjà une grande efficacité aux initiatives heureuses que fait naitre une reprise d'intérét envers les richesses naturelles, culturelles et commerciales qu'abrite le Saint-Laurent.

Il reste donc à structurer et à animer l'action des intervenants de toutes sources.

A cet égard, la Société de Développement Economique du Saint-Laurent était créée en novembre 1985. Cette chambre de commerce du Saint-Laurent québécois s'est dotée de plusieurs comités sectoriels dont un sur le tourisme et les loisirs reliés à la présence du Saint-Laurent. Ce comité permet la jonction des forces vives du milieu et facilite la concertation de tous les acteurs.

Ce n'est que par un travail acharné et suivi que, tous ensemble, nous pourrons contribuer à une mise en valeur récréo-touristique de ce patrimoine unique qu'est le SaintLaurent: f

\section{Rutbrence}

(1) Quebec, Conseil exdcutif, Le Saint-Laurant, ressource nationale pribritaire: rapport du projet Saint-Laurent, Qubbec: Ministare des Communtcations, 1965, $191 \mathrm{p}$. 